\title{
Lesion Revascularisation Subsequent to Femoropopliteal Spot Stenting Using the Multi-LOC Stent Delivery System
}

\author{
MARTIN SIGL $^{1}$, KLAUS AMENDT $^{2}$, MATTHIAS WALISZEWSKI $^{3,4}$ and NILS RATHMANN ${ }^{5}$ \\ ${ }^{1}$ First Department of Medicine, Universitätsmedizin Mannheim (UMM), Mannheim, Germany; \\ ${ }^{2}$ Department of Angiology and Cardiology, Diakonissenkrankenhaus Mannheim, \\ Gefäßzentrum Oberrhein, Mannheim, Germany; \\ ${ }^{3}$ Medical Scientific Affairs, B. Braun Melsungen AG, Berlin, Germany; \\ ${ }^{4}$ Department of Internal Medicine and Cardiology, \\ Charité Universitätsmedizin, Campus Virchow, Berlin, Germany; \\ ${ }^{5}$ Institute of Clinical Radiology and Nuclear Medicine, \\ Universitätsmedizin Mannheim (UMM), Mannheim, Germany
}

\begin{abstract}
Background/Aim: Restenosis and stent fractures are well-characterised treatment failures following femoropopliteal (FP) stent-based interventions. We aimed to determine patterns of restenosis and fracture rates following focal stenting of FP arteries. Patients and Methods: This retrospective study investigated angiographic patterns of restenosis and the occurrence of stent fractures following focal FP stenting with the multiple stent delivery system VascuFlex ${ }^{\circledR}$ Multi-LOC. Results: We identified 10 patients with 10 (of 129) FP lesions (mean length $11.7 \pm 4.6 \mathrm{~cm}$ ) and a total of 51 (of 646) Multi-LOC stents that underwent clinically driven target lesion revascularizations (TLR) after $11.5 \pm 9.2$ months, due to symptomatic recurrence of stenosis. None of the Multi-LOC stents had fractured. No isolated instent restenosis (>50\%) was observed. Median diameter lumen loss was significantly more pronounced at the interstent segments (27.0\%) compared to in-stent segments $(7.8 \%$, $p=0.023)$. Conclusion: No evidence of fracture or susceptibility to stent-related restenosis using Multi-LOC stents was observed. Focal FP stenting may be more effective when combined with strategies known to reduce restenosis in non-stented artery segments.
\end{abstract}

This article is freely accessible online.

Correspondence to: Martin Sigl, First Department of Medicine, Universitätsmedizin Mannheim (UMM), Theodor-Kutzer-Ufer 1-3, 68167 Mannheim, Germany. Tel: +49 6213833046, Fax: +49 6213831970, e-mail: martin.sigl@umm.de

Key Words: Femoropopliteal lesions, restenosis, spot stenting, stent fracture, target lesion revascularization.
To date, restenosis following stent-based femoropopliteal (FP) interventions is an unsolved complication despite numerous efforts to improve patency rates. As the population with FP interventions continues to increase, binary stenosis has become a frequent consequence.

In contrast to long-segment ('full metal jacket') stent-based FP interventions (1), angiographic restenosis patterns of TLR and the frequency of stent fractures following FP "spotstenting" have not been evaluated. A previous study has demonstrated higher patency rates of spot-stenting compared to long-segment stenting following (subintimal) recanalization of chronic total FP occlusions (2). A multi-centre randomized study comparing primary long versus short stent coverage of FP lesions (PARADE II; ClinicalTrials.gov-NCT02701881) is currently recruiting patients.

Our study aimed to determine both patterns of restenosis and fracture rates following focal stenting of FP arteries, using $13 \mathrm{~mm}$ long Nitinol stents. Analogous to preclinical results (3), we hypothesised that the short Multi-LOC stent was not susceptible to stent fracture and/or in-stent restenosis.

\section{Patients and Methods}

Study cohort. The study cohort consisted of 10 consecutive patients that had target lesion revascularizations (TLR) following FP focal stenting using the VascuFlex ${ }^{\circledR}$ Multi-LOC stent system (B.Braun Melsungen, Germany). At the time of the index procedure, FP lesions had been focally stented using this stent system as a bailout following angioplasty of symptomatic de novo lesions. Both the index procedures and the TLR were performed at a high-volume vascular centre, the Gefäßzentrum Oberrhein, Diakonissenkrankenhaus Mannheim in Germany, a teaching hospital of the University of Heidelberg, between September 2015 and January 2019. The data were retrospectively retrieved from medical charts and electronic medical records. All patients of the study cohort had initially been entered in the 


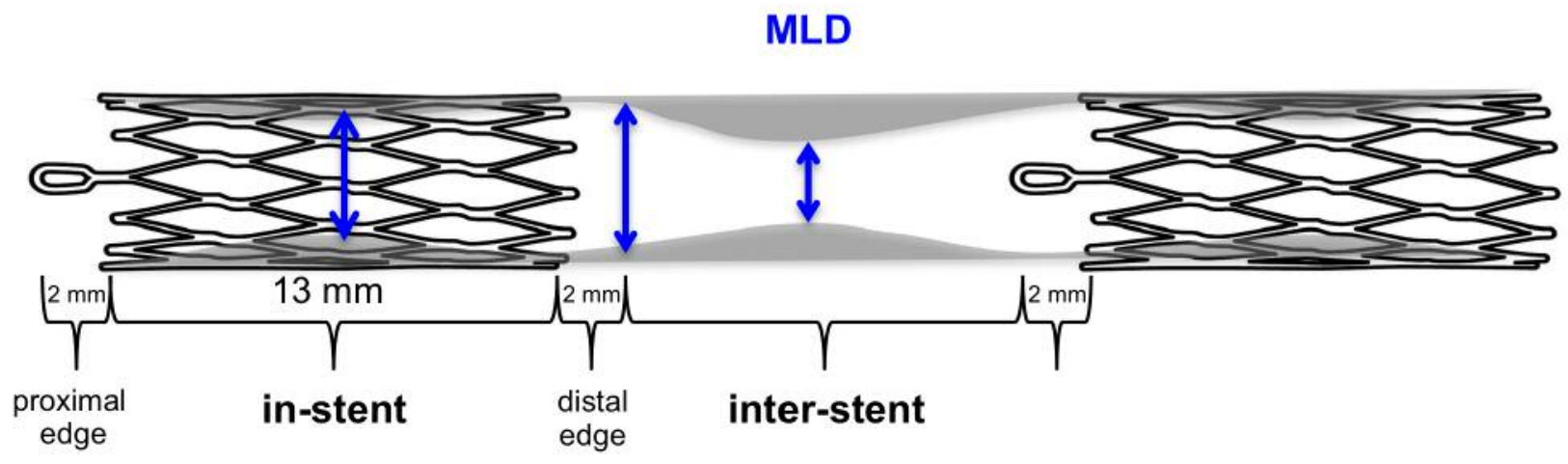

Figure 1. Schematic diagram of quantitative vascular measurements. To determine the pattern of restenosis, the minimal lumen diameter (MLD) was measured along the target lesion at predefined sites. According to the manufacturer's instructions for use, a minimum distance of 5 mm between the Multi-LOC stents is recommended.

LOCOMOTIVE all-comers registry (ClinicalTrials.govNCT02900274), the initial data from which have been published previously $(4,5)$. Briefly, the VascuFlex ${ }^{\circledR}$ Multi-LOC system used in this trial is a new generation 6-French multiple stent delivery system with six self-expanding nitinol stents mounted on one over-the-wire (0.035 inch) catheter, developed for provisional stenting of the femoropopliteal artery in case of insufficient acute results following balloon dilatation.

TLR was defined as clinically-driven re-intervention performed for restenosis of $>50 \%$ diameter stenosis by angiography within the original target lesion and was demonstrated by a decrease in the Rutherford scale by at least one category. TLRs were evaluated and documented by clinical pre-procedural technical examination.

The present study complied with the Declaration of Helsinki (6) and was approved by the local ethics committee, Medical Ethics Commission II, Faculty of Medicine Mannheim, University of Heidelberg, Germany (ID 2019-1117R). Informed consent was obtained from all individual participants included in the study. All data were analysed anonymously. Data protection was in accordance to the European Union Data Protection Directive (7).

Study Endpoints. The primary endpoints of this analysis were i) the characterisation of binary restenosis pattern and ii) the prevalence of Multi-LOC stent fractures. All images and lesion characteristics, including the lesion length, occurrence, pattern and degree of restenosis, and the existence of stent fractures were analysed by two interventional radiologists of the Department of Clinical Radiology and Nuclear Medicine, University Medical Centre Mannheim, Germany. Both reviewers had experience in performing and scientifically evaluating vascular interventions for many years, and they evaluated all angiograms in a blinded fashion using the radiology information system (RIS) and the picture archiving and communication system (PACS; Syngo ${ }^{\circledR}$ PACS, Siemens Medical Solutions). The reviewers were not involved in the development or performance of the evaluated Multi-LOC focal stenting procedure.

Stent fractures. Images to evaluate stent fractures were acquired, using the stented limb segment placed parallel to the X-ray receptor (Artis zee, Siemens Healthineers, Erlangen, Germany) for best visualisation of the individual struts throughout each stent procedure. A minimum of two different projections were evaluated.
High magnification of the images of each single stent was used. A stent fracture was defined as a clear interruption of stent struts identified on X-ray from multiple projections.

Restenosis. All follow-up and index (post-procedural) angiograms were reviewed and the pattern of restenosis was classified as follows: i) in-stent-restenosis, ii) proximal and distal edgerestenosis, and iii) "inter-stent restenosis". The restenosis pattern of two of the lesions with acute thrombotic occlusions was analysed following local lysis therapy only and before further dilatation. Edge stenosis was defined as $>50 \%$ stenosis within the 2 -mm proximal or distal segment from the stent edge, including the stent margin. The distance of $2 \mathrm{~mm}$ was chosen based on the manufacturer's instructions for using the VascuFlex ${ }^{\circledR}$ Multi-LOC device, recommending a minimum distance of $5 \mathrm{~mm}$ between the implanted stents. Stenoses occurring between the stents, but outside this range, were defined as "inter-stent" restenosis (Figure 1).

The length of the expanded Multi-LOC stents $(13 \mathrm{~mm})$ was used for calibration in the quantitative vascular analysis. In order to minimize the measurement bias due to different angiographic projection angles, only anterior-posterior projections ( 0 degree) were used for quantitative analysis. A reference lumen diameter (RLD) was calculated as the mean of the proximal and distal RLD of every target lesion. The minimal lumen diameter (MLD) was measured along the target lesion at predefined sites: i) MLD within the 2-mm proximal segment from the stent edge in each individual stent including the stent edge, ii) MLD within each stent, iii) MLD within the 2-mm distal segment from the stent edge in each individual stent including the stent edge, and iv) MLD between stents ("inter-stent"). Diameter stenosis (DS) was calculated, as follows:

\section{$\mathrm{DS}=\mathrm{MLD} / \mathrm{RLD}$}

The degree of restenosis (diameter lumen loss) in corresponding locations at the index-angiograms and re-angiograms were calculated as follows:

$$
\text { delta-DS }=\left(\mathrm{MLD}_{\mathrm{Index}}-\mathrm{MLD}_{\mathrm{TLR}}\right) / \mathrm{RLD} \times 100
$$

Negative diameter lumen "loss" values indicated a lumen gain of stented vessel segments. 
Table I. Patient characteristics at the time of the index-intervention and $T L R$.

\begin{tabular}{|c|c|c|}
\hline & Index-intervention & TLR \\
\hline Patients, $\mathrm{N}$ & \multicolumn{2}{|c|}{10} \\
\hline Male, $\mathrm{N}$ & \multicolumn{2}{|c|}{9} \\
\hline Age, years, mean $\pm S D$ & $74.1 \pm 8.5$ & $75.1 \pm 8.7$ \\
\hline \multicolumn{3}{|l|}{ Rutherford classes, $\mathrm{n}$} \\
\hline 2 (moderate) & 3 & 3 \\
\hline 3 (severe) & 7 & 7 \\
\hline $\begin{array}{l}\text { Maximal walking distance } \\
\text { in treadmill testing* prior to } \\
\text { intervention, metre, mean } \pm \mathrm{SD}\end{array}$ & $108 \pm 71$ & $94 \pm 41$ \\
\hline $\begin{array}{l}\text { Ankle-brachial index** prior } \\
\text { to intervention, mean } \pm \mathrm{SD}\end{array}$ & $0.62 \pm 0.22$ & $0.70 \pm 0.28$ \\
\hline $\begin{array}{l}\text { Chronic renal insufficiency } \\
(\mathrm{GFR}<60 \mathrm{~mL} / \mathrm{min}), \mathrm{n}\end{array}$ & 2 & 2 \\
\hline End-stage renal disease, $\mathrm{n}$ & 0 & 0 \\
\hline Diabetes mellitus, $\mathrm{n}$ & 6 & 6 \\
\hline Hypertension, $\mathrm{n}$ & 9 & 9 \\
\hline Hyperlipidaemia, n & 5 & 5 \\
\hline Active nicotine consumption, $\mathrm{n}$ & 10 & 10 \\
\hline Platelet aggregation inhibitor, $\mathrm{n}$ & 10 & 10 \\
\hline Statin, $\mathrm{n}$ & 10 & 8 \\
\hline
\end{tabular}

Data are given as mean \pm SD or $\mathrm{n}(\%)$. *All patients with claudication performed treadmill testing at a treadmill velocity of $3.5 \mathrm{~km} /$ hour and on an incline of $12 \%$. **There were no patients with incompressible arteries due to severe mediasclerosis. SD: Standard deviation; GFR: glomerular filtration rate.

Follow-up. Patients with vascular history were re-assessed through physical examination in 6 month-intervals. The ankle-brachial index and the Rutherford clinical classes (8) were determined at all follow-up intervals. Duplex ultrasound (MyLab70, Esaote, Milan, Italy) was also performed for morphologic and functional evaluation of the femoropopliteal axis with significant restenosis defined as peak-velocity-ratios $\geq 2.4$ at the target lesion site.

Statistics. Prior to our analysis, we performed a sample size calculation, aiming to assess at least 50 Multi-LOC stents for subsequent statistical analysis. A mean number of 5.1 released stent segments per patient was estimated, based on values among the first 75 patients of the LOCOMOTIVE registry (5). Therefore, we expected to analyse 10 lesions subjected to repeat angioplasty.

Demographic and clinical characteristics of the study population are reported as means and standard deviations (SD) or as medians and interquartile ranges for continuous variables, according to their distribution. Categorical data are given as counts and percentages. The Pearson's chi-squared test was used to analyse categorical variables. The Mann-Whitney $U$-test was applied to determine significant differences between non-parametric continuous variables (diameter stenosis at different locations). All statistical analyses were performed again after adjusting for the effect of lumen gain. $p$-Values $<0.05$ were considered statistically significant All statistical analyses were performed using SPSS version 23 (IBM, Munich, Germany).
Table II. Patient characteristics at the time of the index-intervention and $T L R$.

\begin{tabular}{|c|c|c|c|}
\hline & Index-intervent & tion & TLR \\
\hline FP lesions treated, $\mathrm{n}$ & \multicolumn{3}{|c|}{10} \\
\hline Stents analysed, $\mathrm{n}$ & \multicolumn{3}{|c|}{51} \\
\hline FP lesion length, mm, mean \pm SD & \multicolumn{3}{|c|}{$11.7 \pm 4.6$} \\
\hline Lesion length stented, $\%$ & \multicolumn{3}{|c|}{$56 \pm 3$} \\
\hline FP lesion location, $\mathrm{n}$ & & & \\
\hline Proximal or mid SFA & \multicolumn{3}{|c|}{7} \\
\hline Distal SFA or PA & \multicolumn{3}{|c|}{5} \\
\hline Severe calcification, $\mathrm{n}$ & \\
\hline Total occlusion, $\mathrm{n}$ & \multicolumn{3}{|c|}{$\begin{array}{ll} & 10 \\
3 \text { (Chronic) } & 2 \text { (Acute thrombotic) }\end{array}$} \\
\hline \multicolumn{4}{|l|}{ Reference vessel diameter } \\
\hline for balloon and stent selection, $\mathrm{n}$ & 5 & & 5 \\
\hline $\begin{array}{l}5 \mathrm{~mm} \\
6 \mathrm{~mm}\end{array}$ & 5 & & 5 \\
\hline \multicolumn{4}{|l|}{ Tibial run-off, $\mathrm{n}$} \\
\hline 1 -vessel & 3 & & 3 \\
\hline 2-vessel & 3 & & 4 \\
\hline 3-vessel & 4 & & 4 \\
\hline \multicolumn{4}{|l|}{$\begin{array}{l}\text { Approach (common } \\
\text { femoral artery), } \mathrm{n}\end{array}$} \\
\hline Antegrade & 9 & & 9 \\
\hline Retrograde & 1 & & 1 \\
\hline \multicolumn{4}{|l|}{ Balloon dilatation, $\mathrm{n}$} \\
\hline POBA & 10 & & - \\
\hline DCB & - & & 10 \\
\hline \multicolumn{4}{|l|}{$\begin{array}{l}\text { Multi-LOC stents implanted } \\
\text { per lesion, } n\end{array}$} \\
\hline
\end{tabular}

FP: Femoropopliteal; SFA: superficial femoral artery; PA: popliteal artery; SD: standard deviation; POBA: plain old balloon angioplasty; DCB: drug-coated balloon.

\section{Results}

From institutional experience with Multi-LOC Device within the first four years since its introduction and its use in patients, 119 cases with 129 FP lesions were treated using 646 implantations of single 13-mm long Multi-LOC stents. We identified 10 patients with $10 \mathrm{FP}$ lesions and a total of 51 MultiLOC stents that received TLR due to symptomatic recurrence of stenosis. The patient characteristics are summarised in Table I. At the time of the index-intervention, all FP lesions had been pre-dilated using plain old balloon angioplasty (POBA). TLR was performed using drug-coated balloon (DCB) angioplasty without further stent placement. Lesion characteristics and procedural data are shown in Table II.

The mean time to TLR was $11.5 \pm 9.2$ months. None of the Multi-LOC stents with closed-cell design was fractured. Restenosis $>50 \%$ was localised within the inter-stent segments only, or extended into both the stented and inter-stent segments. None of the 51 stents showed evidence of isolated in-stent restenosis. Figure 2 shows an example of an FP lesion, in 


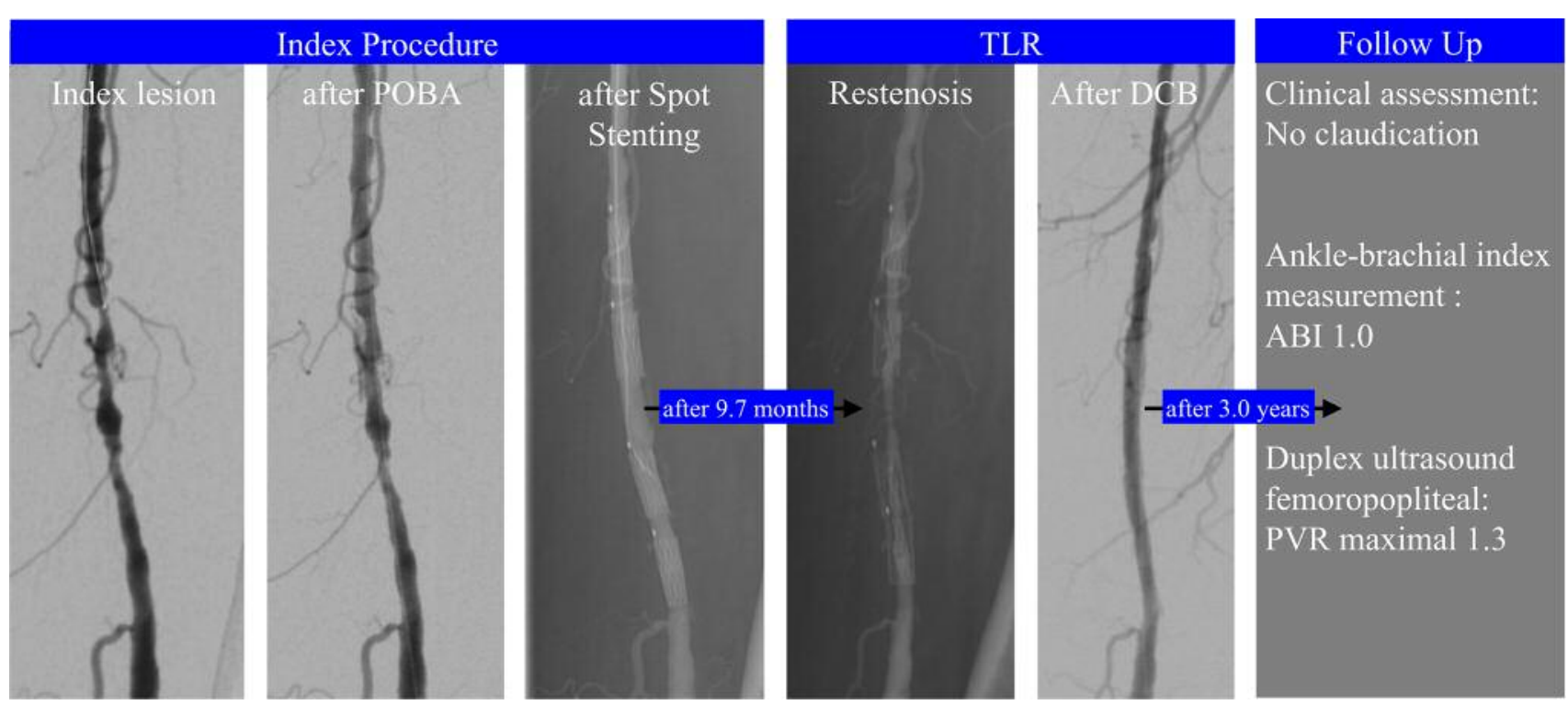

Figure 2. Example of corresponding index and target lesion revascularizations (TLR) angiograms. During the index procedure the 8.4-cm long stenotic lesion of the left distal superficial femoral artery was predilated with an uncoated 5-mm balloon. After deployment of 4 Multi-LOC stents $(5 \times 13 \mathrm{~mm})$ to cover dissections and recoil, post-stent dilatation was performed with a balloon of a corresponding diameter to ensure complete development and axial stent alignment. After 9.7 months clinically driven TLR was performed. The angiogram showed restenosis both in the interstent and the stented segments. Drug-coated balloon angioplasty was performed. Follow-up examinations including colour duplex ultrasonography measuring peak systolic velocity ratio (PVR), and ankle-brachial index (ABI) measurement did not show any restenosis for 3 years following TLR.

which TLR was performed together with the corresponding index angiograms.

Within a median follow-up period of 35.7 months (interquartile range 27.1 to 37.7 months) following TLR, 6 of the lesions showed no signs of recurrent target lesion restenosis, 2 patients had clinically-driven re-TLR within 12 months (one of these reported drug non-compliance), and in 2 additional patients restenosis occurred at 34.0 and 36.8 months following TLR.

Diameter lumen loss at the time of TLR was significantly more pronounced at the inter-stent segments compared to the in-stent segments (median diameter stenosis $27.0 \%$ vs. $7.8 \%$, $p=0.023$ ). A statistically significant difference continued to exist after gating in-stent lumen gain of some stented vessel segments. The measurement data at different sites are summarised in Figure 3.

\section{Discussion}

Recently, we published results from the multi-centre LOCOMOTIVE Study using the Multi-LOC system in complex FP lesions, and found, the all-cause TLR rate was $9.3 \%$ in the overall cohort at 12 months (5). In this singlecentre study, we analysed TLR lesions following FP MultiLOC stenting in a targeted manner.

The study cohort represented a "negative selection" of patients with spot-stented FP lesions. First, according to the
LOCOMOTIVE study design, a stent-based strategy was chosen as bailout at the time of the index procedure. Reasons for bailout stenting were i) elastic recoil and/or haemodynamically relevant dissection after pre-dilation of severely calcified stenotic lesions and ii) chronic total occlusions, two of the most challenging subsets of FP disease. With an average of 5.1 Multi-LOC stents implanted per lesion, over half of the index lesion lengths (mean 11.7 $\mathrm{cm}$ ) had been stented. Three of 10 lesions were chronic total occlusions. Second, the patient cohort analysed in this study underwent TLR due to clinically relevant restenosis. All 10 patients continued smoking and the majority presented hypertension and diabetes mellitus, while two patients stopped taking statins after the index procedure. These factors may have contributed to the occurrence of restenosis.

The main findings of this study confirmed our hypothesis that Multi-LOC stents are not susceptible to fracture or stent-related restenosis but result in significantly more pronounced lumen loss in the inter-stent segments. The stents may be too short $(13 \mathrm{~mm})$ to experience relevant biomechanical forces relative to fractures and might not be susceptible to myointimal hyperplasia because of reduced chronic trauma to the vessel wall (short stent length and no oversizing).

While restenosis is a common issue following FP interventions, the mechanisms leading to binary stenosis differ greatly among the different primary revascularisation 


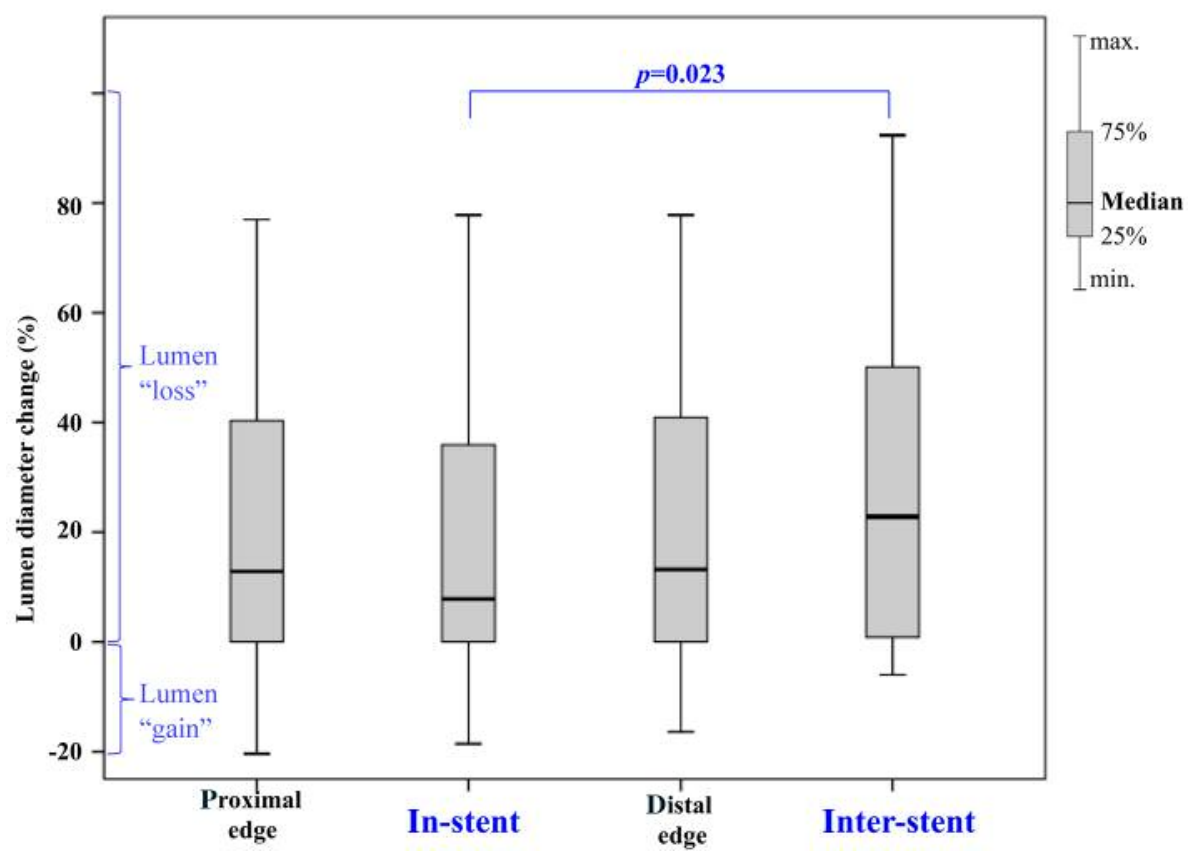

Figure 3. Restenosis pattern. Lumen diameter changes assessed by quantitative vascular analysis. The values indicate the loss (positive values) or gain (negative values) of diameter stenosis from the index procedures (final angiograms) to the follow-up angiograms prior to TLR along the target lesion at the predefined measurement sites.

and scaffolding techniques. Generally, inflammatory reactions of the vessel wall and neointimal proliferation can occur as a result of mechanical dilatation secondary to balloon treatment, either with or without stent deployment (9). Following stenting, foreign body interaction with the vessel wall could lead to stent fatigue and may compromise the integrity of the vessel wall, exacerbating and prolonging the proliferative response, and leading to myointimal hyperplasia and binary stenosis. Regarding restenosis following focal stenting using the Multi-LOC stent device, angiographic follow-up evaluation should be performed to address specific features of this "spot-stenting" technique. While the target lesions were pre-dilated over the entire length (in our TLR cohort with plain-old balloon angioplasty only), stenting should provide vessel scaffolding selectively in lesion segments with high-grade dissections and/or recoil, according to the interventional philosophy to leave as little metal behind as possible. As a result, only the stented segments are subject to chronic outward force of the self-expanding stents and can both increase the in-stent volume during the following months (10) and/or exacerbate the proliferative vessel response, which can lead to in-stent restenosis. Our study allowed the assessment not only of luminal narrowing (negative vascular remodelling resulting from neointimal hyperplasia) but also of a slight luminal enlargement of some of the stented artery segments (positive lumen gain). Both effects seemed to play a minor role in our study cohort. This may be partly due to the fact that stent oversizing was avoided, which is known to promote neointimal proliferation and restenosis (11).

The absence of stent fractures in this study was in accordance with preclinical findings in the porcine model, where short stents did not fracture in actively bended arterial segments compared to longer standard nitinol stents, similar in total length to the multi-stented artery segment (3). Short self-expanding nitinol stents of only $13-\mathrm{mm}$ in length might be too short to experience relevant mechanical stress (e.g. flexion forces). Importantly, stent fracture rates vary between different types of stents. For example, the 12-month fracture rates of 2 cohorts employing the same stent was $3.1 \%$ for lesions averaging $7.1 \mathrm{~cm}$ in the RESILIENT study (12) compared to $27.1 \%$ for lesions averaging $11.8 \mathrm{~cm}$ reported in the TIGRIS study BMS arm (13). The mean lesion length of $11.7 \mathrm{~cm}$ in our study cohort was similar to the latter study.

Furthermore, using finite element analysis, it has recently been shown that physiological deformation and atherosclerotic plaques, both of which are frequent issues in FP lesions, significantly contribute to early fatigue in stents (14). Numerous efforts have been made to produce "fracture-free" stents able to withstand the rigorous mechanical forces in the FP segment. However, it remains questionable whether improved stent-design could resolve all of the issues associated with foreign body-vessel-interaction. Although the consequences of stent fractures are not fully understood and 
are inconsistently reported in the literature, the discussion of stent fracture cannot be ignored. In any case, preventing fullmetal coverage means minimizing the issues of foreign body-vessel-interactions. In a perfused human cadaver model, none of seven different longer FP stents was able to accommodate all FP deformation modes without either restricting or exacerbating baseline deformations within or outside the stented segment (15). In contrast, the influence of multiple short stents on the inter-stent segments is largely unknown and future characterisation of the biomechanical and haemodynamic consequences of focally stented FP lesions is warranted.

The Tack Endovascular System ${ }^{\mathrm{TM}}$ (Intact Vascular, USA), developed to fix dissecting membranes following balloon angioplasty, also aims to provide spot treatment with less metal implantation. The small nitinol rings (6-mm in length) have yielded good results in short (mean length $5.1 \mathrm{~cm}$ ) and less calcified lesions (graded severe in only $5.4 \%$ ) in the TOBA II trial, with a TLR rate of $10.5 \%$ at 12 months (16). However, due to their lower scaffolding capacity, defined as a lower intended radial force, they may not be suitable for severe vessel responses of recoil and dissections following balloon dilation of severely calcified lesions.

Analogous to preclinical results (3), this study confirms that Multi-LOC stents are not susceptible to in-stent restenosis. In summary, we conclude that the short stents cause little chronic trauma to the vessel wall. Problems mainly arise proximal and distal of the stents. First, in severely calcified lesions, acute flow-limiting dissection or recoil after pre-dilation can barely be mechanically stabilised outside the stents. Both vessel preparation techniques, such as vessel scoring or debulking, as well as prolonged balloon inflation times, have the potential to significantly reduce the rate of dissection $(17,18)$. While the overall concept of spot stenting has yielded some promising preliminary results, the size of a "spot" and the optimal stent length for an individual lesion are unknown. In some cases, stents longer than $13 \mathrm{~mm}$ might be warranted for provisional stenting, still avoiding a "full-metal jacket". Second, compared to POBA, the benefits of DCB in reducing late lumen loss due to myointimal hyperplasia, binary restenosis, and TLR in FP lesions are well known (19). In our cohort, none of the initial angioplasties had been performed with DCB. In contrast, our follow-up observations after DCB angioplasty showed a long-lasting effect following TLR of spot-stented FP lesions within a three-year period. Even though DCB angioplasty has emerged as the mainstay of therapy for FP lesions (20), many interventionalists perform plain angioplasty without drug release as the standard of therapy for FP lesions, one of the reasons being the limited reimbursement of the higher costs and some recently published safety concerns of Paclitaxel eluting devices.

Finally, the results of the LOCOMOTIVE Extended Study (ClinicalTrials.gov-NCT02900274) should provide further insights concerning the risk factors for TLR following focal stenting of FP lesions.

There are several limitations in our case series. This was an explorative retrospective study of a small and heterogeneous group of symptomatic patients receiving FP TLR. At the time of the index procedure, pre-dilation was performed exclusively using POBA without additional vessel preparation. Furthermore, half of the lesions involved the distal superficial femoral and/or popliteal arteries, which have traditionally been, albeit not in our view, incomprehensively considered as no stent zones. With regards to quantitative analysis, digital subtraction angiography has some shortcomings. As a "luminography" technique, it provides little information about vessel wall morphology and the MLD may be misleading in cases of eccentric stenosis. Due to our efforts to minimise intervention time, radiation, and contrast medium exposure, we avoided different angiographic projection angles. As a consequence, only anterior-posterior projections (0 degree) were available for the quantitative analysis.

Taken together, our data showed that neither stent fractures nor stent-related restenosis seem to play a decisive role in TLR following focal stenting of complex FP lesions with the Multi-LOC stent delivery system. Based on these findings and the follow-up observations after TLR, we hypothesise that focal FP stenting might be even more effective when combined with strategies to optimise plaque development aimed at reducing restenosis in non-stented artery segments, such as i) vessel scoring, ii) debulking, iii) prolonged balloon inflation times, and iv) the use of drugcoated-balloons. Future research can help to further optimize the spot stenting strategy.

\section{Conflicts of Interest}

KA is a co-inventor of a patent application for the Multi-LOC device used in this study and has received lecturer honoraria from B. Braun to conduct this study. MW is a full-time employee in the Medical Scientific Affairs department of B. Braun Melsungen AG, Vascular Systems, Berlin/Germany. MS and NR have no conflicts of interest to declare.

\section{Authors' Contributions}

MS prepared the initial manuscript and designed the study. MS and KA performed the treatment. MW analysed data. NR made a decisive contribution to completion of the manuscript. KA, MW, and NR provided critical revisions for intellectual content. All authors approved the final version.

\section{Acknowledgements}

We acknowledge financial support by Deutsche Forschungsgemeinschaft within the funding program Open Access Publishing, by the Baden-Württemberg Ministry of Science, Research and the Arts, and by Ruprecht-Karls-Universität Heidelberg. 


\section{References}

1 Tosaka A, Soga Y, Iida O, Ishihara T, Hirano K, Suzuki K, Yokoi $\mathrm{H}$, Nanto $\mathrm{S}$ and Nobuyoshi M: Classification and clinical impact of restenosis after femoropopliteal stenting. J Am Coll Cardiol 59(1): 16-23, 2012. PMID: 22192663. DOI: 10.1016/j.jacc.2011.09.036

2 Hong SJ, Ko YG, Shin DH, Kim JS, Kim BK, Choi D, Hong MK and Jang Y: Outcomes of spot stenting versus long stenting after intentional subintimal approach for long chronic total occlusions of the femoropopliteal artery. JACC Cardiovasc Interv 8(3): 472480, 2015. PMID: 25703873. DOI: 10.1016/j.jcin.2014.10.016

3 Sigl M, Dudeck O, Jung J, Koelble H and Amendt K: Multiple stent delivery system, Multi-LOC, a new technology for spotstenting of the femoropopliteal artery - proof of concept study in a preclinical large animal model. Vasa 46(6): 446-451, 2017. PMID: 28825354. DOI: 10.1024/0301-1526/a000657

4 Amendt K, Beschorner U, Waliszewski M, Sigl M, Langhoff R, Thalwitzer J, Redlich U, Vogel B, Härtel D and Zeller T: First clinical experience with the Multi-LOC multiple stent delivery system for focal stenting in long femoro-popliteal lesions. Vasa 46(6): 452-461, 2017. PMID: 28854856. DOI: 10.1024/0301-1526/a000658

5 Sigl M, Beschorner U, Zeller T, Waliszewski M, Langhoff R, Tautenhahn $\mathrm{J}$ and Amendt K: Focal stenting of complex femoropopliteal lesions with the Multi-LOC Multiple Stent Delivery System: 12-Month Results of the Multicenter LOCOMOTIVE Study. Cardiovasc Intervent Radiol 42(2): 169175, 2019. PMID: 30361959. DOI: 10.1007/s00270-018-2095-9

6 World Medical Association Declaration of Helsinki: Ethical principles for medical research involving human subjects. World Medical Association. JAMA 310: 2191-2194, 2013.

7 Regulation (EU) 2016/679 of the European Parliament and of the Council of 27 April 2016 on the protection of natural persons with regard to the processing of personal data and on the free movement of such data, and repealing Directive 95/46/EC (General Data Protection Regulation). OJ119(1), 2016. Available from: https://eurlex.europa.eu/legal-content/EN/TXT/?uri=celex\%3A32016R0679

8 Rutherford RB, Baker JD, Ernst C, Johnston KW, Porter JM, Ahn $\mathrm{S}$ and Jones DN: Recommended standards for reports dealing with lower extremity ischemia: revised version. J Vasc Surg 26(3): 517-538, 1997. PMID: 9308598. DOI: 10.1016/s07415214(97)70045-4

9 Costa MA and Simon DI: Molecular basis of restenosis and drug-eluting stents. Circulation 111(17): 2257-2273, 2005. PMID: 15867193.

10 Miki K, Fujii K, Shibuya M, Fukunaga M, Imanaka T, Kawai K, Tamaru H, Sumiyoshi A, Nishimura M, Horimatsu T, Saita T, Yoshihara N, Kimura T, Honda Y, Fitzgerald PJ, Masuyama T and Ishihara $\mathrm{M}$ : Impact of stent diameter on vascular response after self-expanding paclitaxel-eluting stent implantation in the superficial femoral artery. J Cardiol 70(4): 346-352, 2017. PMID: 28254383. DOI: 10.1016/j.jjcc.2016.12.011

11 Saguner AM, Traupe T, Räber L, Hess N, Banz Y, Saguner AR, Diehm N and Hess OM: Oversizing and restenosis with selfexpanding stents in iliofemoral arteries. Cardiovasc Intervent Radiol 35(4): 906-913, 2012. PMID: 22006026. DOI: 10.1007/ s00270-011-0275-y

12 Laird JR, Katzen BT, Scheinert D, Lammer J, Carpenter J, Buchbinder M, Dave R, Ansel G, Lansky A, Cristea E, Collins TJ, Goldstein J, Jaff MR and RESILIENT Investigators: Nitinol stent implantation versus balloon angioplasty for lesions in the superficial femoral artery and proximal popliteal artery: twelvemonth results from the RESILIENT randomized trial. Circ Cardiovasc Interv 3(3): 267-276, 2010. PMID: 20484101. DOI: 10.1161/CIRCINTERVENTIONS.109.903468

13 Laird JR, Zeller T, Loewe C, Chamberlin J, Begg R, Schneider PA, Nanjundappa A, Bunch F, Schultz S, Harlin S, Lansky A and Jaff MR: Novel nitinol stent for lesions up to $24 \mathrm{~cm}$ in the superficial femoral and proximal popliteal arteries: 24-month results from the TIGRIS randomized trial. J Endovasc Ther 25(1): 68-78, 2018. PMID: 29285955. DOI: 10.1177/1526602817749242

14 Lei L, Qi X, Li S, Yang Y, Hu Y, Li B, Zhao S and Zhang Y: Finite element analysis for fatigue behaviour of a self-expanding Nitinol peripheral stent under physiological biomechanical conditions. Comput Biol Med 104: 205-214, 2019. PMID: 30529572. DOI: 10.1016/j.compbiomed.2018.11.019

15 MacTaggart J, Poulson W, Seas A, Deegan P, Lomneth C, Desyatova A, Maleckis K and Kamenskiy A: Stent design affects femoropopliteal artery deformation. Ann Surg 270(1): 180-187, 2019. PMID: 29578912. DOI: 10.1097/SLA.0000000000002747

16 Bosiers M, Scheinert D, Hendriks JM, Wissgott C, Peeters P, Zeller T, Brodmann M, Staffa R and TOBA Investigators: Results from the Tack Optimized Balloon Angioplasty (TOBA) study demonstrate the benefits of minimal metal implants for dissection repair after angioplasty. J Vasc Surg 64(1): 109-116, 2016. PMID: 27139789. DOI: 10.1016/j.jvs.2016.02.043

17 Kim TH, Katsetos M, Dahal K, Azrin M and Lee J: Use of rotational atherectomy for reducing significant dissection in treating de novo femoropopliteal steno-occlusive disease after balloon angioplasty. J Geriatr Cardiol 15(4): 254-260, 2018. PMID: 29915614. DOI: 10.11909/j.issn.1671-5411.2018.04.006

18 Horie K, Tanaka A, Taguri M, Kato S and Inoue N: Impact of prolonged inflation times during plain balloon angioplasty on angiographic dissection in femoropopliteal lesions. J Endovasc Ther 25(6): 683-691, 2018. PMID: 30203701. DOI: 10.1177/ 1526602818799733

19 Caradu C, Lakhlifi E, Colacchio EC, Midy D, Bérard X, Poirier $\mathrm{M}$ and Ducasse E: Systematic review and updated meta-analysis of the use of drug-coated balloon angioplasty versus plain old balloon angioplasty for femoropopliteal arterial disease. J Vasc Surg 70(3): 981-995.e10, 2019. PMID: 31126769. DOI: 10. 1016/j.jvs.2019.01.080

20 Aboyans V, Ricco JB, Bartelink MEL, Björck M, Brodmann M, Cohnert T, Collet JP, Czerny M, De Carlo M, Debus S, EspinolaKlein C, Kahan T, Kownator S, Mazzolai L, Naylor AR, Roffi M, Röther J, Sprynger M, Tendera M, Tepe G, Venermo M, Vlachopoulos C, Desormais I and ESC Scientific Document Group: 2017 ESC Guidelines on the Diagnosis and Treatment of Peripheral Arterial Diseases, in collaboration with the European Society for Vascular Surgery (ESVS): Document covering atherosclerotic disease of extracranial carotid and vertebral, mesenteric, renal, upper and lower extremity arteries. Endorsed by: the European Stroke Organization (ESO) the Task Force for the Diagnosis and Treatment of Peripheral Arterial Diseases of the European Society of Cardiology (ESC) and of the European Society for Vascular Surgery (ESVS). Eur Heart J 39(9): 763816, 2018. PMID: 28886620. DOI: 10.1093/eurheartj/ehx095

Received October 9, 2019

Revised October 22, 2019

Accepted October 24, 2019 